\title{
CONDENSATION EFFECTS ON RAYLEIGH SCATTERING MEASUREMENTS IN A SUPERSONIC WIND TUNNEL
}

\author{
b y \\ B. Shirinzadeh, M. E. Hillard, and R. J. Exton \\ Instrument Research Division, NASA Langley Research Center \\ Hampton, VA 23665
}

\begin{abstract}
Rayleigh and Raman scattering measurements have been performed in the 20-inch, Mach 6 wind tunnel at the NASA Langley Research Center. Rayleigh results show signal levels which are much higher than expected for molecular scattering in the tunnel, while densities deduced from spontaneous Raman scattering of molecular nitrogen are in good agreement with the expected nitrogen densities in the facility. The apparent discrepancy in the Rayleigh result is attributed to cluster formation as a result of expansion i $\mathrm{n}$ the tunnel. The dependence of the Rayleigh signal on the stagnation pressure and temperature is also discussed.
\end{abstract}




\section{INTRODUCTION:}

Rayleigh scattering is a technique which has been used as a tool to study total concentration profiles for many years. It has been demonstrated that Rayleigh scattering can be used to obtain two and three dimensional pictures of molecular density in low speed flows. ${ }^{1,2}$ Most recently, this technique has been extended to supersonic flows and two dimensional pictures of flow fields have been obtained. ${ }^{3}$ In supersonic flows, however, due to the cooling which takes place as the gas is expanded through a nozzle, some gas constituents may condense and form clusters. ${ }^{4-7}$ The Rayleigh scattering from cluster formation may interfere with the Rayleigh scattering signal due to molecular nitrogen and oxygen, thus making the interpretation of the data difficult. This paper reports quantitative results on the effect of the nucleation process on the Rayleigh signal in a supersonic wind tunnel. These results were obtained in the free stream of the 20-inch, Mach 6 air tunnel at the NASA Langley Research Center. It will be shown that the Rayleigh signal depends on the stagnation temperature and pressure in a way not accounted for by the isentropic expansion of a perfect gas. This apparent discrepancy can be understood in terms of the strong dependence of the Rayleigh cross section on the size of the cluster. It will be argued that, under our experimental conditions, the extent of this clustering is not sufficient to significantly change the bulk properties of the flow (namely, density and temperature); yet, it can significantly hamper molecular density measurements based on the Rayleigh scattering technique. This argument is further supported by comparing simultaneous flow field measurements of 
signal levels from Rayleigh scattering with the spontaneous Raman scattering of molecular nitrogen.

\section{EXPERIMENTAL:}

Measurements were performed in the free-stream of the 20-inch, Mach 6 wind tunnel at the NASA Langley Research Center. In this tunnel, "dry" a ir is supplied from a reservoir with a pressure of $4.137 \mathrm{MPa}(600 \mathrm{psi})$ and a temperature of $555.2 \mathrm{~K}\left(540^{\circ} \mathrm{F}\right)$. The gas is heated by an electrical resistance heater. An activated alumina dryer provides a dewpoint temperature equal to 232.4 $\mathrm{K}\left(-41^{\mathrm{O}} \mathrm{F}\right)$ at a pressure of $4.137 \mathrm{MPa}(600 \mathrm{psi})$. The gas is then brought to a settling chamber which contains a perforated conical baffle at the entrance and screens at the exit; the maximum pressure is $3.620 \mathrm{MPa}(525 \mathrm{psi})$. It is expanded through a nozzle with the throat dimensions of $.861 \mathrm{~cm}$ by $50.8 \mathrm{~cm}$ into the $52.07 \mathrm{~cm}$ by $50.8 \mathrm{~cm}$ test section. Measurement location was at a distance of $308.20 \mathrm{~cm}$ down stream from the nozzle. There existed no capability for measuring the water concentration either in the settling chamber before the expansion or in the test section while the gas was flowing. Therefore, a $n$ accurate assessment of the water concentration in the test section was not possible. However, If one assumes saturated water vapor pressure at room temperature and at a pressure of $4.137 \mathrm{MPa}$, then an upper limit of $1.4 \times 10^{15}$ molecules $/ \mathrm{cm}^{3}$ for the water concentration in the test section may be deduced.

Figure 1 shows a schematic diagram of the experimental setup which includes both the Rayleigh and the vibrational Raman scattering detection systems. As is depicted in the figure, the frequency-doubled output of a $\mathrm{Nd}$ YAG pulsed laser was used as the light source. This output near $532 \mathrm{~nm}$ was focused at the center of the tunnel using a $40 \mathrm{~cm}$ focal length lens. The 
Rayleigh scattered signal generated along the line was imaged onto a gated, single-intensified CCD camera. The video signal from the camera was digitized using a frame-grabber card and stored in the computer. Similarly, through the opposite window, the scattered radiation which constitutes both Rayleigh and Raman signals was imaged onto the slit of a double monochromator where the Raman component of molecular nitrogen near $607 \mathrm{~nm}$ was resolved from the Rayleigh signal. This Raman signal was then detected by a photomultiplier tube (PMT), processed by a boxcar, digitized using a transient digitizer, and stored in the computer. In this way, one obtains a single-point nitrogen density measurement.

To minimize uncertainties associated with the laser energy fluctuations, a small portion of the beam was split into a photodiode (P.D.) whose output was sampled and stored in the transient digitizer. The output of the digitizer was then transferred into the computer. In this way, Rayleigh and Raman signals were normalized to the laser energy on a per-shot basis.

In Rayleigh scattering experiments, there may exist two sources of background: ambient light and the stray laser light scattered from windows and walls. To minimize the effect of the background room light, the intensifier was gated in time with a duration of 90 microseconds. Although data were taken with the room lights off, this gating helped considerably to ease the alignment procedures and reject the remainder of the background room light. Since in Rayleigh scattering one observes the scattered radiation at the same wavelength as the laser beam, the scattering from windows and walls may present a potential problem. Through baffling, this problem was reduced but not eliminated. In fact, this background constituted about $30 \%$ of 
the full dynamic range of the video signal. For each frame, this background was subtracted by comparing regions in a frame which included Rayleigh signal with those without the Rayleigh signal present.

To minimize uncertainties associated with the photon flux, solid angle of collection, scattering cross section, transmission of the optics, and the gain differences for different pixels in the CCD device, it was necessary to obtain a calibration curve for each pixel. This was done by evacuating the tunnel down to the pressure range of interest and taking data over a range of known static pressures. These calibration curves were obtained each time a $\mathrm{n}$ alignment was performed.

Figure 2 shows a plot of the Rayleigh signal in a row of pixels on the CCD camera as a function of pixel number or position. These data were obtained with the static pressure at $12.26 \mathrm{kPa}$ and the temperature at $297.4 \mathrm{~K}$ in the tunnel (no flow). During the calibration process, we encountered the existence of large particles in the tunnel (the effect of a particle on the Rayleigh signal is shown in Fig. 2). To minimize the effect of particles, pixels with a distinct particle signature were ignored in the data analysis.

Figure 3 shows a plot of the Rayleigh signal from a single pixel as a function of the static pressure in the tunnel (no flow). In this plot, the tunnel pressure was varied from $4.67 \mathrm{kPa}$ to $11.73 \mathrm{kPa}$ at room temperature. Each point represents the average of 100 measurements (10 seconds of data) where the standard deviation of the mean value is depicted as the size of the point (the width is not meaningful). The line here represents the least-squares fit to the data with a slope of $\left(4.85 \pm 0.16 \mathrm{kPa}^{-1}\right)$. Hence, for each pixel, a calibration curve is obtained where the slope and intercept are deduced from 
the linear least-squares fit. Similar calibration curves were also obtained for the Raman detection system. An example of such a calibration curve is shown in Fig. 4. The values of the slope and intercept obtained from these calibration curves were then used to deduce the density at each point.

To see how well this procedure works for multi-point measurements of the Rayleigh scattering, we have plotted in Fig. 5 the tunnel pressure deduced from the calibration as a function of the pixel number (or position). Here, there was no flow and the static pressure in the tunnel was adjusted to 12.26 $\mathrm{kPa}$ at room temperature. For each pixel, data were acquired for 100 laser shots $(10 \mathrm{sec})$. The points represent the deduced pressure and the error flags represent the statistical uncertainty of the mean value in the deduced pressure for each pixel. It should be pointed out that this uncertainty includes the uncertainty associated with the calibration and the standard deviation of the mean value of the signal for each pixel. It is seen here that the deduced pressures cluster around an average value of $12.11 \mathrm{kPa}$ and no structure larger than the statistical uncertainty is evident from this plot. The uncertainty in the central region varies between 0.27 to $0.67 \mathrm{kPa}$ depending on the pixel. It should be added that similar plots were also obtained during the tunnel operation and again no systematic variation beyond the statistical uncertainty of the measurements was observed.

\section{RESULTS AND DISCUSSIONS:}

Simultaneous measurements of signals due to both the Rayleigh scattering and the spontaneous Raman scattering were performed in the free stream of the 20-inch, Mach 6 facility. Figure 6 shows a plot of the Rayleigh signal as a function of the stagnation pressure $P_{t}$ in the tunnel. In this figure 
the stagnation temperature $T_{t}$ posted next to each data point is the typical operating temperature for a given stagnation pressure in the tunnel. Also, for the sake of comparison, we have shown the expected Rayleigh signal level. This level corresponds to purely molecular scattering at the calculated air density based on isentropic expansion in the tunnel. The dashed curve shows the result of this calculation. As is evident from this plot, the measured signal levels are higher than expected which implies apparent higher densities; only one point matches expected results. Results obtained using the spontaneous Raman scattering of molecular nitrogen, however, indicate good agreement (within the statistical uncertainty of the measurements) between the deduced densities and those computed from isentropic expansion in the tunnel (Fig. 7).

To understand this apparent discrepancy in Rayleigh scattering results, one should realize that the ordinate in Fig. 6 is a measure of the signal level and is not necessarily a measure of the molecular (air) density in the tunnel. The Rayleigh cross section is proportional to the density of scatterers, the light frequency to the fourth power, and particle radius to the sixth power. Analysis of the dependence of Mie scattering on the size of the particle indicates that Rayleigh scattering is a good approximation for clusters with a radius, a, of less than 0.1 micrometer. This corresponds to a size parameter $\mathrm{q}=2$ a/ of about 1 (where is the wavelength of the laser light). ${ }^{8}$ It is this strong dependence of the Rayleigh cross section on the size of the particle which could give rise to the observed signal levels (which are as high as a n order of magnitude above the expected values). Specifically, the observed signal levels from each pixel could be the result of a few large particles near the upper end of the Rayleigh regime ( $a<0.1$ micrometer) or a large number of smaller particles. To elucidate this further, let us assume that the one order 
of magnitude higher signal level $\left(\mathrm{P}_{\mathrm{t}}=2.41 \mathrm{MPa}\right.$ and $\left.\mathrm{T}_{\mathrm{t}}=486 \mathrm{~K}\right)$ observed i $\mathrm{n}$ figure 6 is due to the scattering from one particle imaged on a pixel of the CCD camera. This particle occupies a cylinder with a radius of 25 micrometers (laser beam) and a height of 240 micrometers (pixel size 12 micrometers, magnification of 1/20) in the test section. Thus, the density of these particles would be about $2 \times 10^{6}$ particles $/ \mathrm{cm}^{3}$. Under the condition of $\mathrm{P}_{\mathrm{t}}=2.41 \mathrm{MPa}$ and $\mathrm{T}_{\mathrm{t}}=486 \mathrm{~K}$, the density of air molecules in the test section is about $2 \times 10^{18}$ molecules $/ \mathrm{cm}^{3}$. If we take the radius for air molecules to be about $0.1 \mathrm{~nm}$ and take into account the dependence of the Rayleigh signal on both the density and the radius of particles, then, one order of magnitude higher signal level can be attributed to a particle density of $2 \times 10^{6}$ particles $/ \mathrm{cm}^{3}$ with a radius of about $15 \mathrm{~nm}$. This is an estimated upper limit on the size of the particles.

We next consider whether these particles are always present in the flow or whether they are generated as a result of cooling which takes place in the expansion process. To answer this, data were taken at a fixed stagnation pressure for several stagnation temperatures. Figure 8 shows a plot of the signal level as a function of the inverse of the static temperature in the tunnel (this temperature was computed using isentropic expansion). The actual stagnation temperatures are shown at the top of the figure for reference. The circled points represent the facility operating temperatures of the tunnel at a given stagnation pressure. Also, for the sake of comparison, we have plotted (dashed lines) the expected Rayleigh signal levels for these experimental conditions. It is seen from this plot that, as one increases the temperature, the observed signal level decreases. For example, for a stagnation pressure of 0.69 $\mathrm{MPa}$, the observed signal level decreases as the stagnation temperature increases until it reaches the expected signal at a stagnation temperature of 
about $491.3 \mathrm{~K}$. If we extrapolate the linear portion of these curves to determine the temperature at which they cross the corresponding expected values, we obtain the stagnation conditions where the signal levels approach purely molecular scattering. The data show that, in order to reach this condition at high stagnation pressures, the tunnel must be operated at stagnation temperatures which are higher than the present capability.

We also reversed the above procedure by varying the stagnation pressure at constant stagnation temperature; the results are shown in Fig. 9. This figure is a log-log plot of the observed Rayleigh signal as a function of the stagnation pressure for a stagnation temperature of $477 \mathrm{~K}$. The slope of 3.2 deduced from this plot indicates that the signal level has an almost cubic dependence on the stagnation pressure (the dependence is linear for purely molecular scattering).

In addition to these data, there is the general observation that the scattering is uniform throughout the measurement volume (scattering from individual large particles rarely occurred under flow conditions and was not the source of the large signals). This uniform scattering and the observed dependence on pressure and temperature suggests that the large signals result from small particles at low concentration which are flow generated (probably due to the cooling that occurs in the expansion process) and not present in the stagnation chamber.

The data presented here are not sufficient to make a definitive statement on the molecular content or the size of these clusters. Also it may be argued that in large facilities such as the 20-inch, Mach 6 facility, there could exist uncertainties in species concentration within the flow, for example, 
water or other unknown impurities. According to the facility operating manual, all of our measurements were performed in the gas phase region of the computed liquid-gas phase diagram for air in this tunnel. For a given stagnation pressure, the typical operating stagnation temperature was higher by about $11 \mathrm{~K}$ than the stagnation temperature which corresponds to the liquefaction. In fact, some of our data were taken at stagnation temperatures about $40 \mathrm{~K}$ higher than the normal operating temperatures, and yet we were observing clustering. One might speculate water as the clustering agent. However, if we consider the dewpoint temperature provided by the facility $\left(\left[\mathrm{H}_{2} \mathrm{O}\right] \sim 8 \times 10^{12}\right.$ molecules/cm ${ }^{3}$ in the test section $)$, one may rule out this possibility based on the fact that there would not be enough number of collisions between the nozzle and the measurement region to form clusters of sufficient size to produce the observed signal levels (about an order of magnitude higher than the molecular scattering). On the other hand, if we consider saturated vapor pressure of water at room temperature and at a pressure of $4.137 \mathrm{MPa}$ which corresponds to $\left[\mathrm{H}_{2} \mathrm{O}\right] \sim 10^{15}$ molecules $/ \mathrm{cm}^{3}$ in the test section, then water could become a possible clustering agent. Molecular nitrogen and atomic argon can not form a critical size cluster under these conditions, ${ }^{9,10}$ since, at the lowest operating temperature, the density of these species in the tunnel is less than the density corresponding to the saturated vapor pressure for these species. For dry air, we are left with two possible candidates for cluster formation, i. e. $\mathrm{O}_{2}$ and $\mathrm{CO}_{2}$. The density of $\mathrm{CO}_{2}$ in the test section is about $6 \times 10^{14}$ molecules $/ \mathrm{cm}^{3}\left(\mathrm{P}_{\mathrm{t}}=2.41 \mathrm{MPa}, \mathrm{T}_{\mathrm{t}}=486 \mathrm{~K}\right)$. Consideration of the total number of collisions required to form a $\mathrm{CO}_{2}$ cluster of sufficient size that can produce the observed signal levels indicates that, this molecule does not meet the criterion. These considerations suggest that, the 
most likely candidates for formation of single species clusters are $\mathrm{O}_{2}$ and $\mathrm{H}_{2} \mathrm{O}$ molecules. It should be emphasized that the above discussion is based o $\mathrm{n}$ extremely simplified assumptions and, as was said in the beginning of this paragraph, our data can not distinguish between species and the size of clusters.

\section{CONCLUSIONS:}

We have demonstrated that the use of Rayleigh scattering for gas density measurements in the free stream of a supersonic tunnel can be seriously hampered by flow generated interference. The data indicate that this interference probably results from a nucleation process. In addition, the strong temperature and pressure dependence of this interference (Figs. 8 and 9) makes the interpretation of flow visualization images generated from Rayleigh scattering difficult.

The data also show, however, that the interference can be significantly reduced (and possibly eliminated) by increasing the local temperature at constant pressure or decreasing the local pressure at constant temperature. Unfortunately, the conditions required to eliminate the free stream interference (in the Mach 6 facility where the experiments were performed) can not be met; however, these local conditions may exist in a model flow field - we intend to investigate this in the future.

\section{ACKNOWLEDGEMENTS:}

The authors would like to thank J. L. Dillon and A. B. Blair for their support and cooperation during the tests in the Mach 6 tunnel. Expert mechanical design and assembly of the structure supporting the laser and 
optics by Bill Chambers is also acknowledged. We would also like to thank Ray Gregory for the design and implementation of the control circuit for the data acquisition system. 


\section{REFERENCES:}

1. M. C. Escoda and M. B. Long, "Rayleigh Scattering Measurements of the Gas Concentration Field in Turbulent Jets," AIAA Journal 21, 81(1983).

2. B. Yip, D. C. Fourguette, and M. B. Long, "Three-Dimensional Gas Concentration and Gradient Measurements in a Photoacoustically Perturbed Jet," Appl. Opt. 25, 3919(1986).

3. M. Smith, A. Smits, and R. Miles, "Compressible Boundary-Layer Density Cross Sections by UV Rayleigh Scattering," Opt. Lett. 14, 916(1989).

4. P. P. Wegener and L. M. Mark, "Condensation in Supersonic and Hypersonic Wind Tunnels," Advances in Applied Mechanics, Vol. V, H. L. Dryden and Th. von Karman, eds., Academic Press, 1958, p 307-447.

5. J. A. Clumpner, J. Chem. Phys. 55, 5042(1971).

6. M. B. Frish and G. Wilemski, "Optical Studies of Condensation Dynamics in a Supersonic Nozzle," Atmospheric Aerosols and Nucleation; Proceedings of the Twelfth International Conference, Vienna, Austria, Aug. 22-27, 1988 (A8942876 18-47). Berlin and New York, Spring-Verlag, 1988, p. 527-530.

7. J. V. Becker, "Results of Recent Hypersonic and Unsteady Flow Research at Langley Aeronautical Laboratory," J. Appl. Phys. 21, 619(1950).

8. C. F. Bohren and D. R. Huffman, "Absorption and Scattering of Light by Small Particles," Wiley-Interscience Publication, John Wiley \& Sons, 1983, p. 130.

9. P. P. Wegener, "Nucleation of Nitrogen: Experiment and Theory," J. Phys. Chem. 91, 2479(1987).

10. B. C. Wu, P. P. Wegener, and G. D. Stein, "Homogeneous nucleation of argon carried in helium in supersonic nozzle flow," J. Chem. Phys. 69, 1776(1978). 


\section{FIGURE CAPTIONS:}

Figure 1. Schematic diagram of the experiment.

Figure 2. CCD signal level as a function of the pixel number.

Figure 3. Typical Rayleigh scattering calibration curve for a pixel on the CCD camera.

Figure 4. Typical calibration curve for the spontaneous Raman scattering of molecular nitrogen.

Figure 5. Plot of the deduced pressure as a function of the pixel number or position.

Figure 6. Rayleigh signal in the wind tunnel as a function of the stagnation pressure.

Figure 7. Comparison between deduced density from Raman scattering of $\mathrm{N}_{2}$ and expected air density.

Figure 8. Rayleigh signal as a function of the inverse of the static temperature.

Figure 9. Rayleigh signal versus stagnation pressure at a constant stagnation temperature of $477 \mathrm{~K}$. 
